\title{
Radical singularities and the future of Pharmacology
}

\section{Pani}

E-mail: lucapani@unimore.it

Challenges and opportunities follow one another when it comes to launching a new on-line scientific journal in the era of internet, flooded - as we all are - by data and in turn drowning in an ocean of ignorance. All more challenging if the daunting task pertains to the Official Journal of one of the oldest and most respected Pharmacological Society in the world such as the Italian one.

And yet, if the history of man could also be narrated through the striving to overcome one's own limits, to move ever further the boundary between what is (a deadly disease) and what it could be (a definite cure), for tens of centuries drugs were at the epicenter of such tentative and most likely they still will be for a long time. Indeed, the global pharmaceutical drug market which was worth about 1,000 billion (= 1 trillion) USD in 2018 has steadily grown with a CAGR* between 4 and $5 \%$ during the recent past and projected to continue to do so forecasting to be worth $\$ 1,170.4$ billion by 2021 . Aging populations (the number of over 65-year-olds is expected to increase from 92 million in 2013 to 148 million in 2060), the growing prevalence of chronic diseases (metabolic syndrome, diabetes and dementia), the appearance of new diseases and the re-emergence of those that were believed to have been eradicated (including measles), climate change, antimicrobial resistance, and of course cancers of various nature will drive the diagnostic and therapeutic research and development $(R \& D)$ in the next decade.

PharmAdvances strategic positioning in the abundant literature offering, is deeply rooted within the tradition of the Scientific Society it represents and with the visionary intention of covering all areas in the complex and articulated R\&D drug product journey from early design to market access; with coming to grips with true innovative pharmacological treatments, often much more expensive than ever before, and with patients' right to timely access to safer and more effective drugs. Outstanding advances in our knowledge of the human genome, biotechnology and precision medicine are creating even more ambitious expectations in society and patients towards new and life-saving pharmacological and biotechnological treatments. We all have also the responsibility to recognize, support and promote real innovation, facilitate the procedures to introduce new drugs in the market, remove those which are useless, intervene where economic interests could prevail on real health needs, even when these concern populations that are orphan, smaller, special, or not as "protected" by the public opinion. The industry, in turn, should take the opportunity of interacting with Academia, the health care professionals, the regulators and the payers from the start, so as to design better trials, speed up the development process, reduce the risk of failure and fast-track access to patients in need. We must converge on the endpoints, on the impact on the quality of life and to acquire knowledge on what happens when - for example - the administration of new products is to be interrupted for any reason. Finally, it is necessary to evaluate the safety of their long-term use and their value, taking into account all the profound differences between the various Countries.

The Pharmaceutical Science of the future will be more and more characterized by a strong vocation in fundamental research, providing creative product design and really informative animal models and experimental 
pre-clinical results to guide the rest of the development process up to the clinical practice. This scenario represents a new reality, for which vast areas of knowledge of the past have become obsolete if not frankly inadequate. Based on hard figures, the first step is to recognize the radical change that is affecting the world of medicines with the advent, abrupt and exponential, of the so called "Big Data" revolution. Technological and biomedical progress is constantly producing a large volume of biological and health data undergoing rapid and continuous growth, generating a solid knowledge base that is potentially able to improve health services, thanks to more effective and personalized approaches in the prevention, diagnosis and treatment of illnesses and the possibility of making the distribution of health services more efficient, at the same time supporting innovation and economic growth. Big Data in pharmacology means large collections of information closely linked to the populations that take medicines, such as biometric data (height, weight, pressure, body fat etc.), data concerning the habits of the populations, uniform data on the objectives that are sought from the therapy, including data on side effects, reference data on the natural progress of diseases and data on the duration of the pharmacological response over time. A number of other biological variables, including geographical, are important to establish the effects of medicines and, equally, knowing whether the effect of a medicine can be influenced by non-biological variables in the populations carries significant weight, just as certain lifestyles can actually alter the efficacy of a medicine or modify its safety profile. The analysis of these data is a complex and costly process that can provide an unequalled quantity of information that should be assessed, selected and arranged in hierarchies. Finally, we cannot fail to acknowledge and therefore address, one of the biggest threats for the future of our profession. We are permeated by a humanistic culture that has led our Country to stand out in the world but in the last few years we have faced a cultural drift that tends to exclude technical knowledge of respected Scientific Societies from decision-making processes up to a worrying illiteracy that paradoxically directs the public opinion towards a conservative regression dominated by irrational drifts when facing natural processes such as illness, choices of lifestyles or attitude towards vaccines, drugs and therapies.

We must maintain what Carl Sagan described as the "thought-provoking sense of wonder", which is innate in man and fills children with curiosity and thirst for knowledge, before it loses its momentum for lack of encouragement as we approach adulthood. When science withdraws, giving up its enchantment potential, that opening - no longer protected - is taken over by the germ of pseudo-science, which penetrates and proliferates with daring consequences.

Recent news reports have provided a variety of examples of denial of science and delegitimization of the set of rules and regulation underpinning the credibility of the international scientific community. In spite of the fact that those rules have allowed man to make a great many breakthroughs in several fields of knowledge and to learn about the world and himself, to take care of himself and others, to cure diseases and prevent them, they are now derided or at best ranked at the level of personal opinions.

In view of the public's thirst for knowledge and of the large amount of more or less reliable and verified sources of information (or pseudo-information) available at a click, it is even more necessary for the Italian Society of Pharmacology to ensure a qualified presence in the globalized and complex world of scientific communication. We must promote a more mature awareness in citizens, enabling them to distinguish good from bad information and not to be led astray by charlatans and conspiracy theories. This is precisely what PharmAdvances intends to do.

Luca Pani, MD

Editor in Chief

${ }^{*}$ CAGR $=$ Composite Annual Growth Rate 